\title{
INDICADORES E CONDIÇÕES ASSOCIADAS AO ENVELHECIMENTO BEM-SUCEDIDO: REVISÃO INTEGRATIVA DA LITERATURA
}

Maria das Graças Melo Fernandes ${ }^{1}$, Antonia Oliveira Silva², Lara de Sá Neves Loureiro², Ana Claúdia Torres Medeiros ${ }^{3}$

\begin{abstract}
RESUMO: O objetivo deste estudo foi identificar, por meio de revisão integrativa da literatura, o conhecimento científico produzido entre 2003 e 2010 sobre indicadores e condições associadas ao envelhecimento bem sucedido. Para esta revisão foram selecionados vinte artigos. Quanto ao país de origem dos autores, observou-se predomínio de estudos desenvolvidos por brasileiros (60\%), seguido de norteamericanos (25\%). Com relação ao tipo de periódico, houve predominância daqueles de caráter multidisciplinar (50\%), com observância de enfermeiros entre os autores, seguido de médicos (30\%) e psicólogos (20\%). Com relação ao nível de evidência dos artigos, a maioria (45\%) apresentava evidência quatro. O conhecimento produzido revelou que o envelhecimento bem sucedido é um fenômeno determinado por condições positivas de natureza biopsicossocial, cultural e espiritual, sendo evidenciado por bom estado de saúde, capacidade funcional física, mental e social, autonomia, senso de autoeficácia, autoestima e satisfação com a vida. PALAVRAS-CHAVE: Envelhecimento; Idoso; Promoção da saúde; Enfermagem.

\section{INDICATORS AND CONDITIONS ASSOCIATED WITH SUCCESSFUL AGING: AN INTEGRATIVE LITERATURE REVIEW}

\begin{abstract}
: the objective of this study was to study, by means of an integrative literature review, the scientific knowledge produced between 2003 and 2010 concerning indicators and conditions associated with successful aging. Twenty articles were selected for the review. Concerning authors' origin, it was noted that $60 \%$ of the articles were written by Brazilians, followed by North Americans ( $25 \%)$. Concerning the type of periodical, those of multidisciplinary character predominated $(50 \%)$ with nurses foremost among the authors, followed by doctors (30\%) and psychologists (20\%). Concerning the level of evidence referred to in the articles, the majority $(45 \%)$ presented level four evidence. The knowledge produced revealed that successful aging is a phenomenon which is determined by positive bio-psychosocial, cultural and spiritual conditions, and is evidenced by a good state of health, functional physical, mental and social abilities, autonomy, a sense of self-efficacy, self-esteem and satisfaction with life. KEYWORDS: Aging; Elderly; Health promotion; Nursing.

\section{INDICADORES Y CONDICIONES ASOCIADAS AL ENVEJECIMIENTO BIEN SUCEDIDO: REVISIÓN INTEGRATIVA DE LA LITERATURA}

RESUMEN: El objetivo de este estudio fue identificar, por medio de revisión integrativa de la literatura, el conocimiento científico producido entre 2003 y 2010 sobre indicadores y condiciones asociadas al buen envejecimiento. Para esta revisión fueron seleccionados veinte artículos. Acerca del país de origen de los autores, se ha observado predominio de estudios desarrollados por brasileños $(60 \%)$, seguido de norteamericanos $(25 \%)$. Sobre el tipo de periódico, hubo predominancia de aquellos de carácter multidisciplinar (50\%), con observancia de enfermeros entre los autores, seguido de médicos (30\%) y psicólogos (20\%). Acerca del nivel de evidencia de los artículos, la mayoria (45\%) presentava evidencia cuatro. El conocimiento producido ha revelado que el envejecimiento bien sucedido es un fenómeno determinado por condiciones positivas de naturaleza biopsicosocial, cultural y espiritual, siendo evidenciado por buen estado de salud, capacidad funcional física, mental y social, autonomía, sentido de autoeficacia, autoestima y satisfación con la vida.

PALABRAS-CLAVE: Envejecimiento; Anciano; Promoción de la salud; Enfermería.

${ }^{1}$ Enfermeira. Doutora em Sociologia. Professora do Departamento de Enfermagem Clínica e do Programa de Pós-Graduação em Enfermagem da Universidade Federal da Paraíba - UFPB.

${ }^{2}$ Enfermeira. Doutora em Enfermagem. Professora do Departamento de Enfermagem em Saúde Pública e Psiquiatria e do Programa de Pós-Graduação em Enfermagem da UFPB.

${ }^{3}$ Enfermeira. Mestranda em Enfermagem pelo Programa de Pós-Graduação em Enfermagem da UFPB.

Autor correspondente:

Recebido: $17 / 05 / 11$

Maria das Graças Melo Fernandes

Aprovado: $20 / 07 / 11$

Universidade Federal da Paraíba

R. Engenheiro Normando Gomes de Araújo, 132 - 58037-125 - João Pessoa-PB-Brasil

E-mail: graacafernandes@hotmail.com 


\section{INTRODUÇÃO}

O envelhecimento populacional aponta no século XXI como importante evento sociodemográfico, com repercussões políticas, econômicas e sociais para os países desenvolvidos e, sobretudo, para aqueles em desenvolvimento. Nestes, o evento ocorre de forma mais acelerada, demandando, assim, a importância de se garantir aos idosos as condições necessárias para que estes alcancem não somente uma maior sobrevida, mas, também, uma "boa velhice". Prolongar a juventude e retardar a morte têm sido ideais permanentes do ser humano. A velhice não se caracteriza apenas por declínio e perdas, mas abriga muitas oportunidades de mudança positiva e de um funcionamento produtivo.

No processo de envelhecimento, o ritmo de declínio das funções orgânicas varia de um órgão a outro, como também entre idosos de mesma idade ${ }^{(1)}$, observação que suscita as seguintes reflexões: o processo intrínseco que ocorre com o avançar da idade, por si só, determina esse declínio ou fatores extrínsecos a esse processo podem exercer papel preponderante nessa diversidade de efeitos? Que estilos de vida, atitudes, ações, valores e condições são necessários para que o indivíduo tenha uma velhice bem sucedida?

A literatura pertinente salienta que o envelhecimento normal se classifica em envelhecimento usual ou comum, no qual fatores extrínsecos intensificam os efeitos desse fenômeno, e envelhecimento bem sucedido ou saudável, no qual tais fatores não estariam presentes ou, quando existentes, seriam de pequena importância no incremento da senescência ${ }^{(2)}$. Essa dicotomia na compreensão do envelhecimento decorre da distinção feita pelo paradigma biomédico referente à saúde e à doença.

Vale ressaltar, também, que o conceito de envelhecimento bem sucedido tem forte componente cultural e é referido alternativamente como velhice saudável, ativa e produtiva, e também como boa qualidade de vida. $O$ conceito foi introduzido na Gerontologia a partir dos anos sessenta, especialmente nos países de elevado bem-estar social, trazendo importante mudança ideológica na concepção da velhice e do envelhecimento ${ }^{(2)}$. Posteriormente, o conceito foi refinado por meio de modelos teóricos que hoje são dominantes na análise do fenômeno observada na literatura gerontológica ${ }^{(3-5)}$.

Na perspectiva biomédica ${ }^{(3)}$, o envelhecimento bem sucedido é caracterizado pelo baixo risco para doenças e incapacidades funcionais, atividade e envolvimento social. No modelo psicológico de velhice bem sucedida $^{(4)}$, o fenômeno é visto como dependente do equilíbrio entre a compensação das perdas associadas ao envelhecimento e da otimização das potencialidades individuais mediante ações educativas, médicas, sociais e outras. Uma terceira concepção de velhice bem sucedida, de natureza social, é a que estabelece que as sociedades podem proporcionar oportunidades de envelhecimento bem sucedido ao implementarem recursos externos por meio de políticas públicas ${ }^{(5)}$.

Considerando os aspectos ora abordados, verifica-se que o conceito de envelhecimento bem sucedido transcende os limites biológicos ao explicar as diferentes condições vivenciadas no curso desse processo, valorizando também os aspectos psicológicos e sociais, e a íntima relação entre eles $^{(1)}$. Esse conceito se sustenta no pressuposto de que o processo de envelhecimento é, até certo ponto, modificável, podendo estar associado especialmente à atividade, à satisfação e à preservação da saúde.

Tendo em vista que, apenas recentemente, o conceito de envelhecimento bem sucedido tem sido analisado de forma mais criteriosa e consistente pela comunidade científica, bem como ainda existem significativas lacunas no que concerne à sua conceptualização, em especial ao entendimento de seus indicadores e de seus determinantes, julga-se pertinente analisar os subsídios que a comunidade cientifica traz com relação a esses aspectos do fenômeno.

Assim sendo, este estudo tem o objetivo de identificar, por meio de uma revisão integrativa da literatura, indicadores e condições associadas ao envelhecimento bem sucedido.

\section{METODOLOGIA}

Estudo descritivo, efetivado por meio de uma revisão integrativa da literatura, que teve como propósito responder à seguinte questão norteadora: qual o conhecimento científico produzido sobre indicadores e condições associadas ao envelhecimento bem sucedido? Esse tipo de revisão contribui no processo de sistematização e análise dos resultados, visando à compreensão de um determinado tema a partir de outros estudos independentes ${ }^{(6)}$, sendo operacionalizado em seis etapas ${ }^{(7)}$ : delimitação da questão de pesquisa; estabelecimento de critérios para inclusão de estudos/ amostragem ou busca na literatura; definição das informações a serem extraídas dos estudos; avaliação dos estudos; interpretação dos resultados; e apresentação da síntese do conhecimento.

As estratégias de busca do corpus literário tiveram a finalidade de localizar, por meio do site da Biblioteca Virtual em Saúde (BVS), estudos publicados nas bases 
de dados Literatura Latino-Americana e do Caribe em Ciências da saúde (LILACS), Scientific Eletronic Library Online (SciELO) e Medical Literature Analysis and Retrieval System Online (MEDILINE), no período de 2003 a 2010, que contemplassem em seus títulos e/ou resumos aspectos relativos a indicadores e/ou fatores associados ao envelhecimento bem sucedido e estivessem disponibilizados na íntegra, gratuitamente, na Internet. Para isso, utilizou-se o descritor envelhecimento de forma combinada com a expressão saudável, o que permitiu a identificação de 54 artigos na base de dados LILACS, 41 na base de dados SciELO e 39 na MEDILINE, totalizando 134 publicações.

Após a leitura criteriosa dos títulos e resumos destes artigos, considerando os critérios de pertinência e consistência do conteúdo, 53 foram excluídos da amostra pela inadequação à questão norteadora, 12 eram duplicados (encontrados concomitantemente na LILACS e na SciELO) e 29 não puderam ser acessados na íntegra pela via eletrônica do acervo do Sistema de Bibliotecas da Universidade, resultando na seleção de 20 artigos que atendiam aos critérios do estudo. Vale destacar que foi acrescida a essa nusca literatura seis estudos, incluindo dois capítulos de livro, que não foram usados na análise dos indicadores e das condições associadas ao envelhecimento bem sucedido, mas utilizados na ancoragem de outros aspectos da pesquisa, a exemplo da problematização e dos procedimentos metodológicos.

Para a coleta dos dados utilizou-se instrumento contendo as variáveis: abordagem metodológica utilizada nos estudos, ano de publicação, origem dos autores, nível de evidência dos estudos e a síntese do conhecimento produzido. Para a definição do nível de evidência dos artigos, foi utilizada a seguinte classificação $^{(8)}$ : nível um: evidências resultantes de metanálise de estudos clínicos controlados e randomizados; nível dois: obtidos em estudos de delineamento experimental; nível três: evidências dos estudos quase experimentais; nível quatro: evidências oriundas de estudos descritivos ou com abordagem qualitativa; nível cinco: evidências provenientes de relatórios de casos ou relatos de experiência; nível seis: evidências baseadas em opiniões de especialista da área.

$\mathrm{Na}$ análise aprofundada dos artigos, efetivada para a identificação do conhecimento produzido, as informações relevantes e pertinentes, conforme julgamento das pesquisadoras, foram sintetizadas em subcategorias que foram incorporadas, conforme suas especificidades, nas categorias preestabelecidas a partir do objeto do estudo: "indicadores de envelhecimento bem sucedido" e "con- dições associadas ao envelhecimento bem sucedido".

\section{RESULTADOS}

Das 20 publicações selecionadas, 9(45\%) eram de natureza quantitativa, 6(30\%) foram abordadas qualitativamente e 5(25\%) compreendiam artigos de revisão. Considerando o ano de publicação, houve predomínio no ano de 2008(35\%) seguido de 2009(20\%). Quanto ao país de origem dos autores, observou-se predomínio de estudos desenvolvidos por autores brasileiros (60\%), seguidos de noteamericanos (25\%). Autores da China, da Inglaterra e da Austrália compõem $15 \%$ dos estudos.

No tocante ao nível de evidência dos artigos, verificaram-se três estudos (15\%) com nível de evidência dois; também 3(15\%), com nível de evidência três; $9(45 \%)$ apresentavam evidência quatro; um artigo (5\%) possuía nível de evidência cinco e, por fim, quatro pesquisas (20\%) expressavam nível seis de evidência. Esses dados revelam que a maioria das pesquisas analisadas se caracterizava como estudo descritivo.

No concernente à síntese do conhecimento produzido pelos estudos analisados relativamente a indicadores e condições associadas ao envelhecimento bem sucedido, esta se encontra disposta na tabela 1.

Tabela 1- Indicadores e condições associadas ao envelhecimento bem-sucedido. João Pessoa, 2010

\section{Indicadores de envelhecimento bem sucedido}

Ausência ou baixo índice de doenças

Percepção positiva do estado de saúde

Capacidade funcional física, mental e social

Índice de massa corporal normal para a idade

Autonomia e senso de autoeficácia

Satisfação com a vida/autoestima preservada

\section{Condições associadas ao envelhecimento} bem sucedido

Bom nível de renda

Bom nível educacional

Prática regular de atividade física

Alimentação saudável

Desuso do fumo e do álcool

Adaptação às mudanças relacionadas à idade

Suporte psicossocial

Relações familiares e de amizade

Engajamento ativo com a vida: papeis familiares, lazer, atividades produtivas

Crença religiosa ou espiritual 


\section{DISCUSSÃO}

Considerando a estreita relação entre os indicadores e as condições associadas ao envelhecimento bem sucedido, a análise de tais informações, realizada nos tópicos que se seguem, será apresentada de forma relacionada quando for pertinente.

Vale ressaltar que a saúde, assim como o envelhecimento, representa um fenômeno multidimensional que apresenta características individuais e coletivas e que envolve os aspectos físicos, cognitivos, psicológicos e sociais da natureza humana. Desse modo, o envelhecimento saudável, ou aquele com menor impacto das doenças deve ser promovido a partir da melhoria de todos esses fatores ao longo de toda a vida do indivíduo ${ }^{(2,9-23)}$.

Tão importante quanto o estado de saúde mensurado por avaliações objetivas, assim também como indicador de envelhecimento bem sucedido é o estado de saúde percebido ${ }^{(2,11-13,16-19,24)}$. Considerando essa avaliação, um idoso portador de doenças compensadas pode referir bem-estar e outro, sem comorbidades, porém, vivendo em condições sociais desfavoráveis, pode apresentar uma saúde autoavaliada como ruim. Esta avaliação é feita pelo idoso com base em critérios pessoais, referenciados a valores e expectativas sociais e individuais, bem como a mecanismos de comparação social e temporal ${ }^{(13,16,19)}$. Idosos que avaliam negativamente seu estado de saúde apresentam risco aumentado de todas as causas de mortalidade, em comparação com aqueles que classificam sua saúde como boa ou excelente ${ }^{(2)}$. Além disso, a autoavaliação positiva da saúde constitui um fator particularmente importante de satisfação de vida ou do bem-estar subjetivo ${ }^{(12)}$.

Outro importante indicador do envelhecimento bem sucedido constitui a manutenção da capacidade funcional física, cognitiva e social ${ }^{(2,9,13-23,25-26)}$. Ela inclui a habilidade do idoso em executar tarefas físicas, a preservação das atividades mentais e uma situação adequada de integração social, sendo determinada por fatores socioculturais, estado de saúde, alterações próprias do envelhecimento e outros ${ }^{(13,23)}$.

A autonomia do idoso é considerada um indicador de saúde e bem-estar e, portanto, de envelhecimento bem sucedido $^{(10,16-17,19-21,26)}$. Ela traduz a emancipação do idoso em relação ao meio-ambiente, bem como a busca da realização de seus projetos de vida, mantendo a concepção de sua identidade e o senso de autoeficácia. A cognição assume papel de relevância na manutenção da autonomia ${ }^{(1,17)}$.

Todos os aspectos ora discutidos, como saúde, capacidade funcional, autonomia e senso de auto- eficácia guardam estreita relação com as variáveis socioeconômicas, a exemplo da renda e do nível educacional $^{(2,9-11,16-18,21,24,26)}$. Tais condições interferem diretamente no desenvolvimento da consciência sanitária do idoso, na prática de autocuidado e, por sua vez, na qualidade do seu envelhecimento.

Estudos sinalizam também que as características ambientais, a dieta e os hábitos de vida saudáveis, bem como a minimização de exposição aos fatores de risco, a exemplo do fumo, do álcool, da obesidade, do sedentarismo, dentre outros, têm repercussões diretas sobre os aspectos fisiológicos e metabólicos do organismo enve1hecido ${ }^{(1-2,9-11,14,19-26)}$. Considerando, de modo particular, a alimentação, ressalta-se que a produção de radicais livres pode ser aumentada na presença de uma alimentação pouco saudável. O consumo excessivo de gorduras, alimentos refinados e o baixo consumo de frutas e hortaliças são hábitos amplamente prejudiciais ao organismo ${ }^{(19)}$.

As modificações estruturais e funcionais observadas no envelhecimento, associadas ao sedentarismo, aceleram o declínio da capacidade funcional, facilitando tanto o desenvolvimento de doenças crônicodegenerativas não transmissíveis como incapacidade física no idoso ${ }^{(10)}$. A prática regular da atividade física promove maior fixação de cálcio nos ossos, aumenta a força e a endurance musculares, o equilíbrio e a flexibilidade, ajuda no controle da glicemia, melhora o perfil lipídico, reduz o peso corporal, melhora a função pulmonar e circulatória e auxilia o controle da pressão arterial. Vale salientar que os benefícios desta prática não se restringem à melhora da condição física, mas favorecem, também, o bem-estar psicológico ${ }^{(11,14)}$.

Entre as condições associadas ao envelhecimento bem sucedido foi apontada por uma quantidade substancial de estudos a importância de o idoso se adaptar positivamente às mudanças relacionadas à idade ${ }^{(1-2,11-13,17,19)}$. Os autores salientam que a competência adaptativa é emocional, considerando as estratégias e habilidades com as quais o indivíduo lida com os fatores estressantes e é também cognitiva, no tocante à capacidade para resolução de problemas, e comportamental, no referente ao desempenho social. A capacidade de adaptação frente às mudanças próprias da idade, que são diferentes de uma pessoa para outra, compreende um processo individual e diferenciado em relação às variáveis mentais, comportamentais e sociais ${ }^{(1)}$.

A literatura aponta o engajamento social vivenciado pelo idoso como, possivelmente, um dos maiores preditivos da velhice bem sucedida ${ }^{(2,10-11,13,16,18-19,22,25-26)}$. Afirma, ainda, que a deterioração da saúde pode ser causada 
não somente por um desgaste natural do organismo e exposição a fatores de riscos, mas, também, pela redução da quantidade ou qualidade das relações sociais ${ }^{(2,10)}$. Um dos maiores problemas da pessoa idosa é a solidão, fruto da viuvez, perda de amigos, aposentadoria.

Assim sendo, a capacidade de interagir socialmente, de estar ativo na comunidade, de cultivar amigos, é fundamental para o idoso, a fim de que ele possa conquistar e manter as redes de apoio social, que constituem conjuntos hierarquizados de pessoas que mantêm entre si laços típicos das relações de dar e receber. A estrutura das relações sociais refere-se à organização do vínculo de pessoas umas com as outras, podendo ser descrita sob diferentes aspectos: número de relações e papeis sociais que uma pessoa tem; frequência de contatos com vários membros de uma rede; densidade; multiplicidade e reciprocidade de relações entre os membros de uma rede, dentre outros ${ }^{(16,22)}$.

Considerando-se as dimensões do apoio social, este se configura no formal e no informal. Neste último, de notável significância na determinação do envelhecimento bem sucedido, destaca-se a importância da família na atenção às pessoas idosas, graças ao componente afetivo de suas relações. Ela está envolvida, principalmente, no apoio emocional, instrumental e de informação.

Além do apoio social, o desempenho de atividades como as de lazer são reforços ao sentimento de valor pessoal, ao autoconceito e à autoeficácia ${ }^{(1-2,10-13,16,18-19,22,25)}$. Por meio do lazer, o idoso pode sentir-se útil no seu ambiente social, opondo-se ao isolamento e ao recolhimento ${ }^{(11)}$. Do mesmo modo, o aumento do tempo de vida laboral, não somente como meio de sobrevivência, mas, também, como uma atividade prazerosa e uma forma de inserção social, está fortemente correlacionado com o envelhecimento bem sucedido ${ }^{(13)}$.

Por último, estudos enfatizam a relação positiva entre estado geral de saúde, espiritualidade e bem-estar psicológico $^{(9,14-16,17,19,21,26)}$. A vivência da religiosidade por parte de um grupo de 3.968 idosos, mensurada a partir da frequência a templos ou igrejas ao longo de seis anos, foi associada a menor risco relativo de morte ${ }^{(15)}$.

Considerando os aspectos biocomportamentais ou psicossociais mediante os quais as práticas religiosas promovem a saúde, destacam-se os seguintes: promoção de comportamentos e estilos de vida saudável, a exemplo do desuso do fumo e do álcool; provimento de suporte social (muitas vezes, instrumental), por parte de comunidades religiosas, estimulando o sentimento de intimidade, de auxílio mútuo e de amor fraterno, o que proporciona a sensação de pertença a um grupo e o suporte emocional ${ }^{(14)}$. Existem também evidências ${ }^{(9)}$ de que o enfrentamento do tipo religioso entre os idosos é mais utilizado pelos mais velhos, pelas mulheres (especialmente as viúvas) e por aqueles de menor nível de escolaridade e menor status socioeconômico.

\section{CONSIDERAÇÕES FINAIS}

O presente estudo contribuiu para a identificação dos indicadores e dos fatores associados ao envelhecimento bem sucedido, evidenciando, em especial, que o fenômeno é produto de eventos multidimensionais experimentados pelo indivíduo em todo o seu curso de vida. Cabe destacar que os problemas de saúde enfrentados pelos idosos não se iniciam quando estes atingem sessenta anos, mas, são resultantes, dentre outro fatores, de um acúmulo de experiências passadas, dos cuidados com a saúde, das condições de moradia, educação, alimentação e higiene, da prática de atividade física e das oportunidades perdidas ou aproveitadas.

Apesar do exposto, salienta-se que o estudo apresenta fragilidades metodológicas, particularmente relacionadas ao pequeno número de pesquisas analisadas, bem como a diversidade de métodos e de desenhos envolvidos no desenvolvimento destas pesquisas. Desse modo, recomenda-se a realização de uma investigação mais ampla em que se possa aprofundar a análise, destacandose diferentes métodos utilizados, com instrumentos de medidas objetivas e subjetivas que considerem a experiência do próprio idoso. Ouvir idosos sobre um tema que diz respeito tanto ao seu bem-estar como às condições envolvidas na sua determinação ajuda na compreensão das suas necessidades e demandas com vistas ao alcance do envelhecimento bem sucedido.

Por fim, ressalta-se a importância de se produzir reflexões sobre a atenção social e de saúde dirigida aos idosos saudáveis ou vulneráveis. Ressalta-se, também, a importância do estabelecimento de interlocução interdisciplinar entre estudos de natureza psicológica, social e biomédica, destinados a compreender a velhice bem sucedida, com vistas a melhor delimitação desse conceito na atualidade.

\section{REFERÊNCIAS}

1. Nicolazi MC, Silva JKC, Coelho L, Cascaes AM, Büchele F. Qualidade de vida na terceira idade: um estudo na atenção primária em saúde. Cogitare Enferm. 2009; 4(3):428-34.

2. Ribeiro PCC, Neri AL, Cupertino APFB, Yassuda MS. Variabilidade no envelhecimento ativo segundo gênero, 
idade e saúde. Psicol Estud. 2009;14(3):501-9.

3. Rowe JW, Kahn RL. Successful aging. New York: Pantheon Books; 1998.

4. Baltes PB, Baltes MM. Psychological perspectives on successful aging: the model of selective optimization with compensation. In: Baltes PB, Baltes MM, editores. Successful aging: perspectives from the behavioral sciences. New York: Cambridge Univesity Press; 1990. p. 1-34.

5. Riley M, Riley JW. Age integration and lives of older people. Gerontologist. 1994;34(1): 110-5.

6. Garbin LM, Rodrigues CC, Rossi LA, Carvalho EC. Classificação de resultados de enfermagem (NOC): identificação da produção científica relacionada. Rev Gaúch Enferm. 2009;30(5):508-15.

7. Mendes KDS, Silveira RCCP, Galvão CM. Revisão integrativa: método de pesquisa para a incorporação de evidências na saúde e na enfermagem. Texto \& Contexto Enferm. 2008;17(4):758-64.

8. Stetler CB, Morsi D, Rucki S, Broughton S, Corrigan B, Fitzgerald J, et al. Utilization-focused integrative reviews in a nursing service. Appl Nurs Res. 1998;11(4):195-206.

9. Broekman BF, Niti M, Gwee X, Kua EH. Determinants of successful aging using a multidimensional definition among Chinese elderly in Singapore. Am J Geriatr Psychiatry. 2009;17(5):407-16.

10. Chaves ML, Camozzato AL, Eizinik CL, Kaye J. Predictors of normal and successful aging among urbandwelling elderly Brazilians. J Gerontol B Psychol Sci Soc Sci. 2009;64(5):597-602.

11. Briton A, Shipley M, Singh-Manoux A, Marmot MG. Successful aging: the contribution of early-life and midlife risk factors. J Am Geriatr Soc. 2008;56(6):1098-2005.

12. Reichstadt J, Depp CA, Palinkas LA, Folsom DP, Jeste DV. Building blocks of successful aging: a focus group study o folder adults' perceived contributors to successful aging. Am J Geriatr Psychiatry. 2007;15(3):194-201.

13. Montross LP, Depp C, Daly J, Reichstadt J, Golshan S, Moore D, et al. Correlates of sef-rated successful aging among community dwelling older adults. Am J Geriatr Psychiatry. 2006;14(1):43-51.

14. Pell NM, McClure RJ, Barttlett HP. Behavorial determinants of healthy aging. Am J Prev Med. 2005;28(3):298-304.
15. Deep CA, Jeste DV. Definitions and predictors of successful aging: a comprehensive review of larger quantitative studies. Am J Geriatr Psychiatry. 2006;14(1):6-20.

16. Moraes JFD, Souza VBA. Factors associated with the successful aging of the socially-active in the region metropolitan of Porto Alegre. Rev Bras Psiquiatr. 2005;27(4):302-8.

17. Ramos LR. Fatores determinantes do envelhecimento saudável em idosos residentes em centro urbano: Projeto Epidoso, São Paulo. Cad Saúde Pública. 2003;19(3):793-98.

18. Teixeira INDO, Neri AL. Envelhecimento bemsucedido: uma meta no curso da vida. Psic - USP. 2008;19(1):81-94.

19. Vilarino MAM, Lopes MJM. Envelhecimento e saúde nas palavras de idosos de Porto Alegre. Estud Interdiscip Envelhec. 2008;13(1):63-77.

20. Cupertino APFB, Rosa FHM, Ribeiro PCC. Definição de envelhecimento saudável na perspectiva de indivíduos idosos. Psicol Refl Crit. 2007;20(1):81-6.

21. Patrício KP, Ribeiro H, Hoshino K, Bocchi SCM. O segredo da longevidade segundo as percepções dos próprios longevos. Ciênc Saúde Colet. 2008;13(4):1198-2008.

22. Dillaway HE, Byrnes M. Reconsidering successful aging. J Appl Gerontol. 2009;6(3):702-22.

23. Flood MT, Nies MA. Successful aging: selected indicators in a southern sample. Home Health Care Manag Pract. 2009;22(2):111-5.

24. Fieldler MM, Peres KG. Capacidade funcional e fatores associados em idosos do sul do Brasil: um estudo de base populacional. Cad Saúde Pública. 2008;24(2):409-15.

25. Lima AMM, Silva HS, Galhardoni R. Envelhecimento bem-sucedido: trajetórias de um constructo e novas fronteiras. Interface Comun Saúde Educ. 2008;12(27):795-807.

26. Rosa FHM, Cupertino APFB, Neri AL. Significados de velhice saudável e avaliações subjetivas de saúde e suporte social entre idosos recrutados na comunidade. Rev Bras Geriatr Gerontol. 2009;3(2):62-9. 